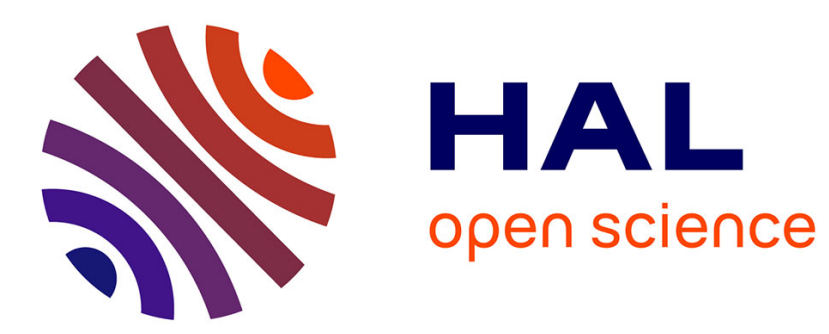

\title{
Improving Spectral Efficiency While Reducing PAPR Using Faster-Than-Nyquist Multicarrier Signaling
}

\author{
Cyrille Siclet, Damien Roque, Alexandre Marquet, Laurent Ros
}

\section{To cite this version:}

Cyrille Siclet, Damien Roque, Alexandre Marquet, Laurent Ros. Improving Spectral Efficiency While Reducing PAPR Using Faster-Than-Nyquist Multicarrier Signaling. International Workshop on Communication Technologies for Vehicles, May 2017, Toulouse, France. hal-01720656

\section{HAL Id: hal-01720656 https://hal.science/hal-01720656}

Submitted on 1 Mar 2018

HAL is a multi-disciplinary open access archive for the deposit and dissemination of scientific research documents, whether they are published or not. The documents may come from teaching and research institutions in France or abroad, or from public or private research centers.
L'archive ouverte pluridisciplinaire HAL, est destinée au dépôt et à la diffusion de documents scientifiques de niveau recherche, publiés ou non, émanant des établissements d'enseignement et de recherche français ou étrangers, des laboratoires publics ou privés. 


\title{
Improving Spectral Efficiency While Reducing PAPR Using Faster-Than-Nyquist Multicarrier Signaling
}

\author{
Cyrille Siclet ${ }^{1}$, Damien Roque ${ }^{2}$, Alexandre Marquet $^{1}$, and Laurent Ros ${ }^{1}$ \\ 1 Univ. Grenoble Alpes, GIPSA-Lab, F-38000 Grenoble, France \\ CNRS, GIPSA-Lab, F-38000 Grenoble, France, \\ \{alexandre.marquet, cyrille.siclet, laurent.ros\}@gipsa-lab.fr \\ 2 Institut Supérieur de l'Aéronautique et de l'Espace (ISAE-SUPAERO) Université \\ de Toulouse, 31055 Toulouse, France \\ damien.roque@isae-supaero.fr
}

\begin{abstract}
Multicarrier modulations are widely used in mobile radio applications due to their adaptability to the time-frequency characteristics of the channel, thus enabling low-complexity equalization. However, their intrinsically high peak-to-average power ratio (PAPR) is a major drawback with regard to implementation issues (e.g., power amplification efficiency, regulatory constraints...).

In this paper, we confirm that the PAPR can be decreased as the signaling density (i.e., spectral efficiency at fixed constellation size) increases, even in the case where symbols cannot be perfectly reconstructed using a linear system. In such a two-dimensional generalization of faster-thanNyquist (FTN) systems, PAPR distribution models from the literature are confirmed by simulation results. Furthermore, for a fixed number of subcarriers, we show that a sufficient condition to yield the optimal PAPR distribution at the output of a critically sampled transmitter is to specify pulse shapes as tight frames. Finally, simulation are performed in the more realistic case of an oversampled transmitted signal.
\end{abstract}

Keywords: Multicarrier modulations, faster-than-Nyquist signaling, peakto-average power ratio, power amplification.

\section{Introduction: PAPR of faster-than-Nyquist multicarrier modulations}

Vehicular communications often imply radio propagation in a mobile multipath environment (e.g., car-to-car communications, miniature unmanned aerial vehicles, etc.). Input-output relation of such a propagation channel usually accounts for time and frequency selectivity [1-3]. In this context, multicarrier modulations can be specified as an approximate eigenstructure of the channel by allocating information symbols to coordinate in the time-frequency plane as illustrated in Fig. $1[4,5]$. A careful design of the multicarrier system (i.e., time-frequency lattice, pulse shapes, etc.) can significantly lower equalization's computational 
complexity compared to more specific waveforms (e.g., single carrier modulations, cyclic-prefixed orthogonal frequency-division multiplexing) [6].

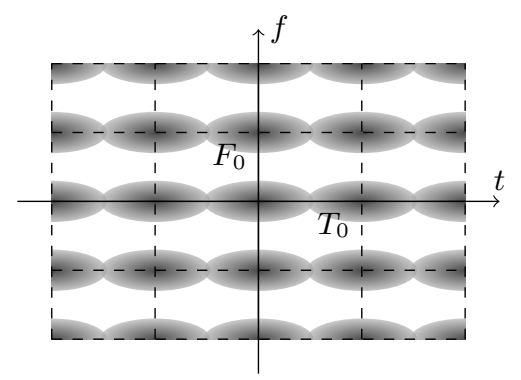

Fig. 1. Representation of a multicarrier signal with a rectangular lattice in the timefrequency plane using Gaussian pulse shapes.

With an increasing need of spectral efficiency, the "faster-than-Nyquist" (FTN) signaling technique has been extended to multicarrier modulations [7]. Denoting $F_{0}$ the inter-carrier spacing and $T_{0}$ the multicarrier symbol duration, the system is referred to as FTN when its density $\rho=1 /\left(F_{0} T_{0}\right)$ is strictly greater than one. Such a constraint implies that transmitted information symbols cannot be perfectly reconstructed using a linear receiver. In other words, for a finite bandwidth and a fixed information symbol constellation of size $N_{c}$, any increase of spectral efficiency $\eta=\rho \log _{2}\left(N_{c}\right)$ beyond $\log _{2}\left(N_{c}\right)$ comes at the cost of interpulse interference that should be mitigated with the help of non-linear receivers $[8,9]$.

Any transmitted multicarrier signal results from a sum of many independent information symbols shaped and modulated onto subchannels of equal bandwidth. It is thus characterized by an intrinsically high peak-to-average power ratio (PAPR) [10]. Since the peak power is usually limited either by regulatory or integration constraints (e.g., linear power amplification), the average power should be adjusted consequently, thus penalizing the link budget.

In this paper, we show that PAPR decreases as the density increases, even in the FTN context $(\rho>1)$, provided that appropriate pulse shapes are used. To this extent, we refer to statistical PAPR models initially assessed for $\rho \leq 1[11$, 12]. We show that a sufficient condition to yield an optimal PAPR distribution with a critically sampled FTN multicarrier transmitted signal is to specify pulse shapes as tight frames. Simulations also reveal the need of more accurate PAPR models in the context of oversampled systems.

The paper is organized as follows. Section 2 presents the multicarrier transmitted signal model considering any density and pulse shape. Section 3 defines PAPR and recalls statistical models from the literature while generating empirical distributions through simulations. The FTN case is extensively investigated in order to emphasize the influence of $\rho>1$ combined with several practical pulse 
shapes sampled at the Nyquist rate or above. Finally, conclusion and insights are given in Section 4 .

\section{System model: (non)-rectangularly shaped multicarrier (FTN) modulations}

Let $K>0$ be the number of multicarrier symbols to be transmitted, and $M>0$ be the number of subcarriers. We define $\left\{c_{m, n}\right\}_{(m, n) \in \boldsymbol{I}} \in \boldsymbol{C}^{K \times M}, \boldsymbol{I}=$ $\{0, \ldots, M-1\} \times\{0, \ldots, K-1\}$ the sequence of zero-mean, independent and identically distributed (i.i.d) information symbols, usually taken in a finite set (or constellation). In the continuous-time domain, the transmitted multicarrier signal is obtained by shaping each symbol by a time-frequency shifted version of a prototype (or pulse shape) $g(t) \in \boldsymbol{R}[13]$ :

$$
s(t)=\sum_{(m, n) \in \boldsymbol{I}} c_{m, n} g_{m, n}(t), \quad t \in \boldsymbol{R}
$$

with

$$
g_{m, n}(t)=g\left(t-n T_{0}\right) e^{j 2 \pi m F_{0} t}
$$

In practical applications, $M$ and $K$ are bounded, $\left\{c_{m, n}\right\}_{(m, n) \in \boldsymbol{I}}$ is square summable and $g(t)$ is also square integrable such that the sum in (1) converges. One recalls that $F_{0}>0$ and $T_{0}>0$ represent elementary symbol spacing, in frequency and time, respectively. By defining the transmission density as $\rho=1 /\left(F_{0} T_{0}\right)$, it can be shown that a necessary condition to perfectly recover the symbols $c_{m, n}$ without interpulse interference (IPI) using a linear receiver is $\rho \leq 1[14$, Chapter 9]. Consequently, such a density parameter can be used to generalize the single carrier Nyquist criterion ${ }^{3}$ to the two-dimensional case (i.e., time-frequency) such that three cases arise [16]:

$-\rho<1$ (or equivalently, $T_{0}>1 / F_{0}$ ) corresponds to a slower-than-Nyquist (STN) or undercritical system;

- $\rho=1$ (or equivalently, $T_{0}=1 / F_{0}$ ) corresponds to a Nyquist rate or critical system;

$-\rho>1$ (or equivalently, $T_{0}<1 / F_{0}$ ) corresponds to a faster-than-Nyquist (FTN) or overcritical system.

Over an additive white Gaussian noise (AWGN) channel, when $\rho \leq 1$, symbols are linearly recovered without IPI and with maximization of the signal-to-noise ratio (SNR) when $\left\{g_{m, n}(t)\right\}_{(m, n) \in \boldsymbol{I}}$ constitutes an orthonormal family, i.e.:

$$
\left\langle g_{m, n}, g_{p, q}\right\rangle=\int_{-\infty}^{+\infty} g_{m, n}^{*}(t) g_{p, q}(t) \mathrm{d} t=\delta_{m, p} \delta_{n, q}
$$

\footnotetext{
3 The Nyquist criterion mentionned herein refers to the interference free condition, as initially stated in [15] in the case of single carrier transmission.
} 
with $\delta$ the Kronecker delta and $(m, n, p, q) \in \boldsymbol{I}^{2}$. On the contrary, when $\rho>1$ ,$\left\{g_{m, n}(t)\right\}_{(m, n) \in \boldsymbol{I}}$ cannot be an orthogonal family and IPI cannot be removed by a linear receiver, but the signal-to-interference-plus-noise ratio (SINR) is maximized when $\left\{g_{m, n}(t)\right\}_{(m, n) \in \boldsymbol{I}}$ forms a tight frame [16]. What is more, according to the Wexler-Raz theorem [14, p. 214], this is equivalent to say that $\tilde{g}_{m, n}(t)$ forms an orthonormal family sharing the same prototype in the dual time-frequency lattice:

$$
\tilde{g}_{m, n}(t)=\sqrt{F_{0} T_{0}} g\left(t-n / F_{0}\right) e^{j 2 \pi m t / T_{0}} .
$$

For numerical purposes, the system may also be described at discrete-time. The transmission generator $g(t)$ is supposed to have a bandwidth $W_{g}$. It results an overall system bandwidth $W=(M-1) F_{0}+W_{g}$ that can be approximated by $M F_{0}$ hereafter assuming $\left|W_{g}-F_{0}\right| /\left(M F_{0}\right) \ll 1$. In practice, it is generally the case if we consider a large number of subcarriers. As a consequence, the signal can be sampled at critical rate $1 / T_{s}=M F_{0}$ and we denote $N$ the number of samples per multicarrier symbol such that $T_{0}=N T_{s}$. Note that the density can be rewritten as $\rho=M / N$ and considering a unique multicarrier symbol, the FTN case is illustrated in the discrete-time domain by a number of samples per multicarrier symbol $N$ less than the number of subcarriers $M$.

Assuming that $g(t)$ has most of its energy in $\left[-T_{s} / 2 ; L_{g} T_{s}-T_{s} / 2[\right.$, the discrete-time transmission prototype can be expressed as

$$
g[k]= \begin{cases}\sqrt{T_{s}} g\left(k T_{s}\right) & \text { if } k \in\left\{0, \ldots, L_{g}-1\right\} \\ 0 & \text { otherwise. }\end{cases}
$$

where the factor $\sqrt{T_{s}}$ is used for energy normalization. From (1), the discretetime transmitted signal can be expressed as

$$
s[k]=\sqrt{T_{s}} s\left(k T_{s}\right)=\sum_{(m, n) \in \boldsymbol{I}} c_{m, n} g_{m, n}[k]
$$

with

$$
g_{m, n}[k]=\sqrt{T_{s}} g_{m, n}\left(k T_{s}\right)=g[k-n N] e^{j 2 \pi \frac{m}{M} k} .
$$

A complete reception stage (including iterative decoding or decision feedback equalization techniques) may be found in [9] and shows that FTN systems may allow an almost perfect recovery of the symbols at an extra computational cost compared to orthogonal linear systems. Such receiving structures won't be detailed hereafter since we focus on the PAPR issue, which only involves the transmitted signal model given in (1) and (6)

\section{Benefits of FTN multicarrier signaling on PAPR}

The PAPR of a signal is defined as the ratio of its peak power and its average power. For a continuous-time multicarrier transmitted signal as defined in (1), 
assumed with a finite time support $[0 ; T]$ with $T \geq T_{0}$, we have:

$$
\mathrm{PAPR}=\frac{\max _{t \in[0, T]}|s(t)|^{2}}{\mathbb{E}\left\{\frac{1}{T} \int_{0}^{T}|s(t)|^{2} \mathrm{~d} t\right\}}
$$

where $\mathbb{E}\{\cdot\}$ is the expectation operator. For a discrete-time (critically sampled) signal as defined in (6), we denote $N_{t}=T / T_{s}$ and we obtain the following expression:

$$
\mathrm{PAPR}_{\mathrm{d}}=\frac{\max _{\left.k \in\left\{0, \ldots, N_{t}-1\right)\right\}}|s[k]|^{2}}{\mathbb{E}\left\{\frac{1}{N_{t}} \sum_{k=0}^{N_{t}-1}|s[k]|^{2}\right\}}
$$

Note that $s[k]$ in the previous relation can be interpolated by a factor $N_{i}$. It is consequently clear that PAPR $\mathrm{d}_{\mathrm{d}}$ approaches PAPR as $N_{i}$ becomes large $\left(N_{i}=4\right.$ is typically sufficient). As discussed in [11], the maximization in (8) or (9) is often performed over a multicarrier symbol duration.

It may be derived from [11] that if

1. $c_{m, n}$ symbols are zero-mean and i.i.d.,

2. $g(t)$ is time-limited,

3. $\sum_{n \in \boldsymbol{Z}}\left|g\left(t-n T_{0}\right)\right|^{2}>0$ for all $t \in \boldsymbol{R}$,

4. $s[k]$ samples are independent from each other,

then, for $M$ large enough (typically, $M \geq 8$ ), the complementary cumulative distribution function (CCDF) of the discrete-time PAPR may be approximated thanks to the formula:

$$
\operatorname{Pr}\left\{\mathrm{PAPR}_{\mathrm{d}}>\gamma\right\} \approx 1-\prod_{k=0}^{N-1}\left(1-e^{-\gamma x[k]}\right)
$$

with

$$
x[k]=\frac{\|g\|^{2}}{N \sum_{n \in \boldsymbol{Z}}|g[k-n N]|^{2}}
$$

and, what is more, the best PAPR performance is achieved when $x[k]=1$, so that we then get

$$
\operatorname{Pr}\left\{\mathrm{PAPR}_{\mathrm{d}}>\gamma\right\} \approx 1-\left(1-e^{-\gamma}\right)^{N} .
$$

Note that the former expression matches traditional (rectangularly shaped) OFDM waveform when $N=M$ (i.e., in the absence of cyclic prefix). Nevertheless, if we manage to satisfy conditions 1 to 4 with an FTN multicarrier system, this PAPR performance will be improved since $\rho>1$ implies $N<M$. Conditions 1 and 2 may obviously be imposed regardless of the system's density. We will now show that condition 3 and 4 are also satisfied if we use tight frames, as recommended in [16] for the sake of SINR maximization.

Indeed, if $g_{m, n}(t)$ (or $g_{m, n}[k]$ for the discrete-time equivalent system), is a tight frame, then the dual family $\tilde{g}_{m, n}(t)=\sqrt{F_{0} T_{0}} g\left(t-n / F_{0}\right) \exp \left(j 2 \pi m t / T_{0}\right)$ 
forms an orthonormal family. This also means that $g(t)$ satisfies the Nyquist criterion at rate $F_{0}$ :

$$
\sum_{m \in \boldsymbol{Z}}\left|\hat{g}\left(f-m F_{0}\right)\right|^{2}=\mathrm{cst},
$$

with $\hat{g}(f)$ the Fourier transform of $g(t)$.

According to [17], (13) implies that the power spectral density of $s[k]$ is white if $c_{m, n}$ are i.i.d and assuming a critical sampling frequency, namely $1 / T_{s}=M F_{0}$. What is more, using the central limit theorem as in [11,12], we get that samples $s[k]$ follow a Gaussian distribution and should be considered as independent within our transmitted signal model (6). What is more, the fact that $g_{m, n}(t)$ forms a tight frame also implies that [14, page 210]

$$
\sum_{n \in \boldsymbol{Z}}\left|g\left(t-n T_{0}\right)\right|^{2}=T_{0}\|g\|^{2}
$$

so that $\sum_{n \in \boldsymbol{Z}}|g[k-n N]|^{2}=N\|g\|^{2}$, and $x[k]$ defined in (11) is therefore equal to 1 .

These results are confirmed by simulations in which we use several prototypes of tight frames: the first one maximizes the time-frequency localization (denoted as TFL) [18], the second one is a square root raised cosine with roll-off equal to $\rho-1$ (denoted as SRRC) and the third one is a rectangular pulse-shape of length $M$ (denoted as RECT-M) or $N$ (denoted as RECT-N). Even if the $M$-samples rectangular window (RECT-M) does not generate a tight frame, it may be shown that the samples of the subsequent multicarrier samples signal are also independent. Nevertheless, in this case, the corresponding $x[k]$ is not a constant, leading to a degraded PAPR performance.

As for the CCDF of the PAPR, we first compare it in the discrete-time case with the theoretical formula given by (12). Figures $2-5$ show a very good match with the theoretical formula whereas the use of a non-tight frame (RECT-M) gives a discrete-time PAPR distribution very different. These simulations also confirm that increasing the number of subcarriers improves accuracy (i.e., the relevance of the central limit theorem).

We then compare the continuous-time PAPR distribution $\left(N_{i}=4\right)$ obtained for the same systems. In this case, the theoretical formula does not hold since signal samples may not be considered independent anymore. Nevertheless, Figures 6-9 show that the relative positions of the different curves are unchanged. Thus, this also confirms that increasing $\rho$ not only increases the spectral efficiency, but also improves the PAPR. In fact, for a given number $M$ of subcarriers, the distribution of the PAPR is the same as the one of an orthogonal system using $M / \rho \leq M$ subcarriers. 


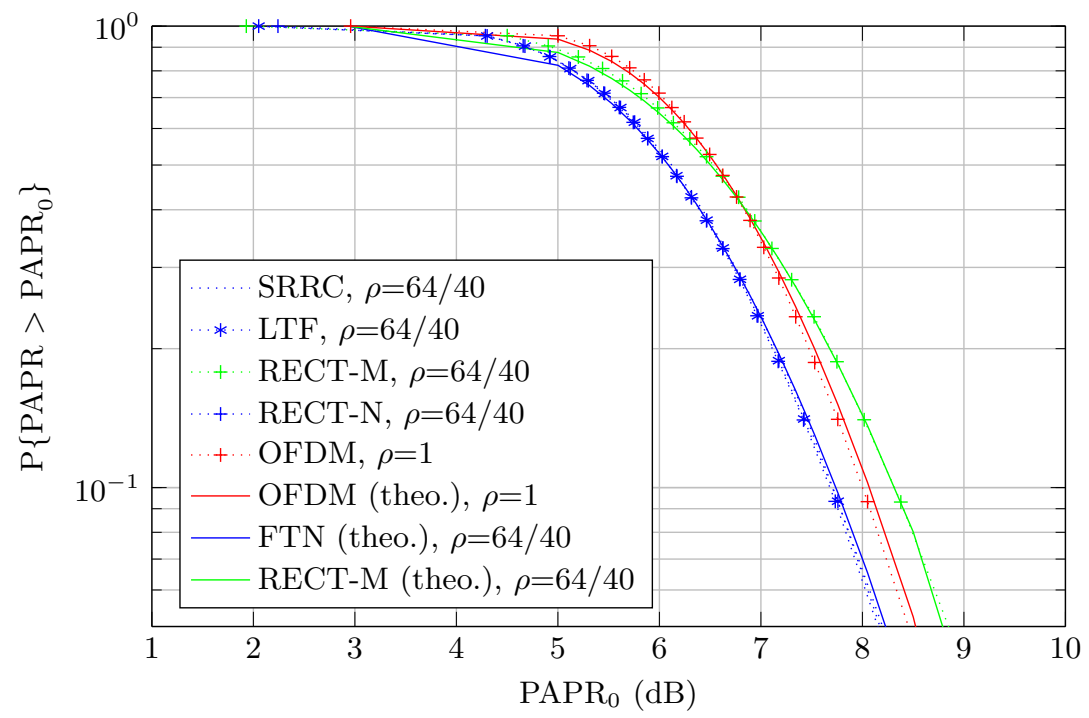

Fig. 2. Discrete-time PAPR comparison for $M=64$, a quadrature phase shift keying (QPSK) constellation, $\rho=1.6$, and various prototypes.

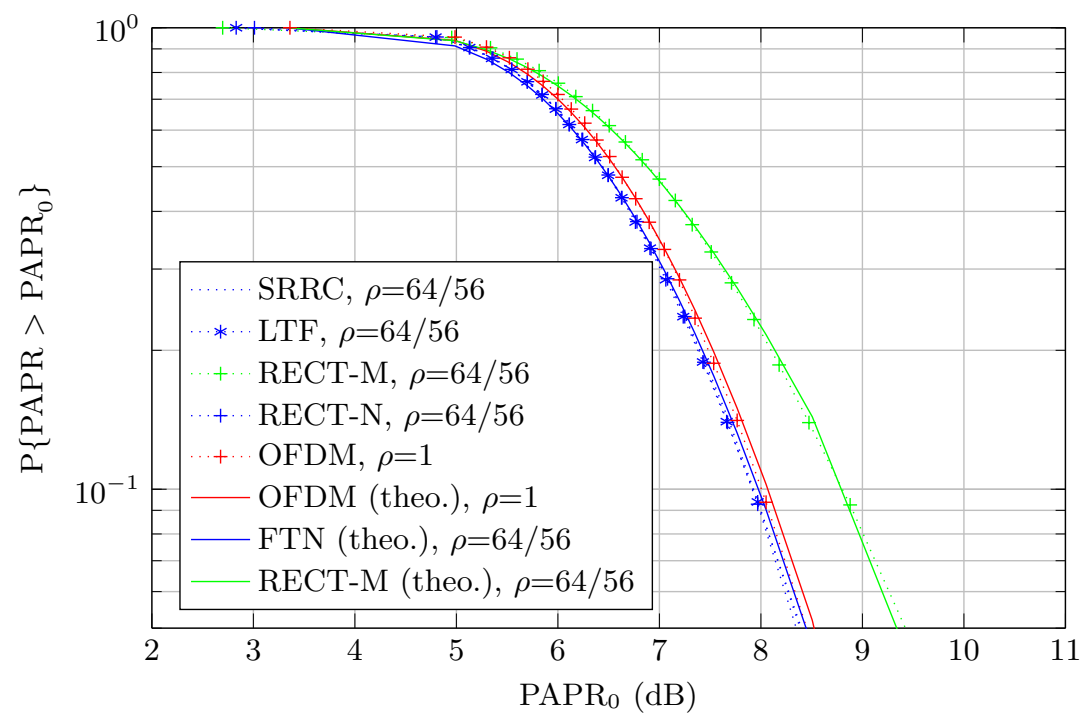

Fig. 3. Discrete-time PAPR comparison for $M=64$, a quadrature phase shift keying (QPSK) constellation, $\rho \approx 1.14$, and various prototypes. 


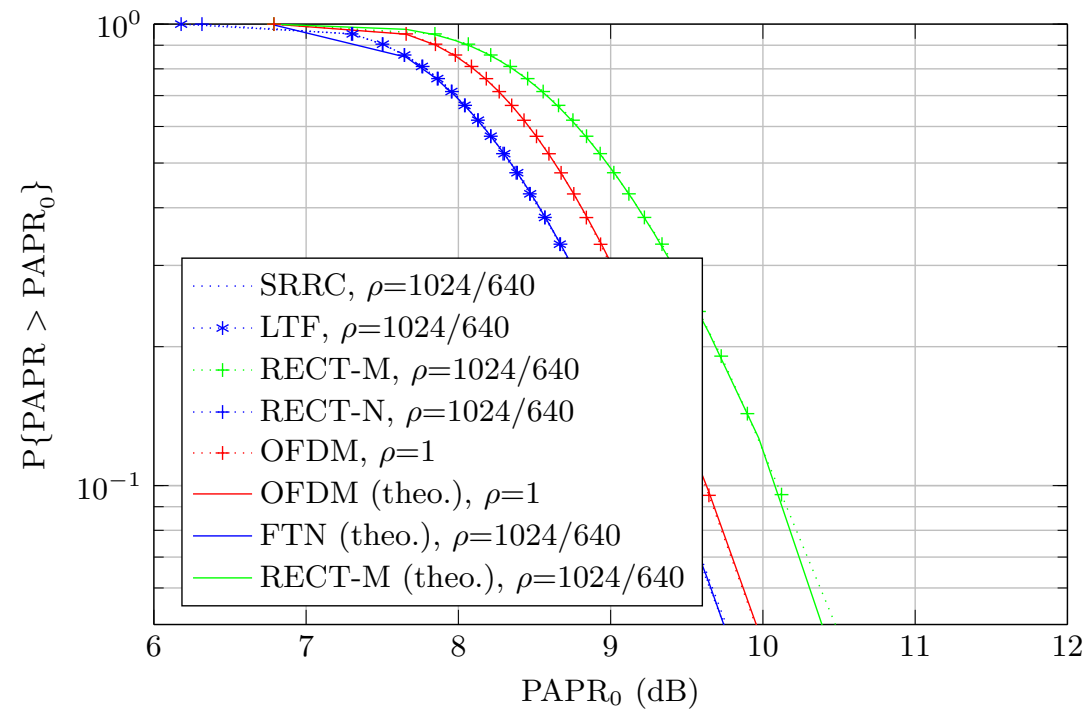

Fig. 4. Discrete-time PAPR comparison for $M=1024$, a quadrature phase shift keying (QPSK) constellation, $\rho=1.6$, and various prototypes.

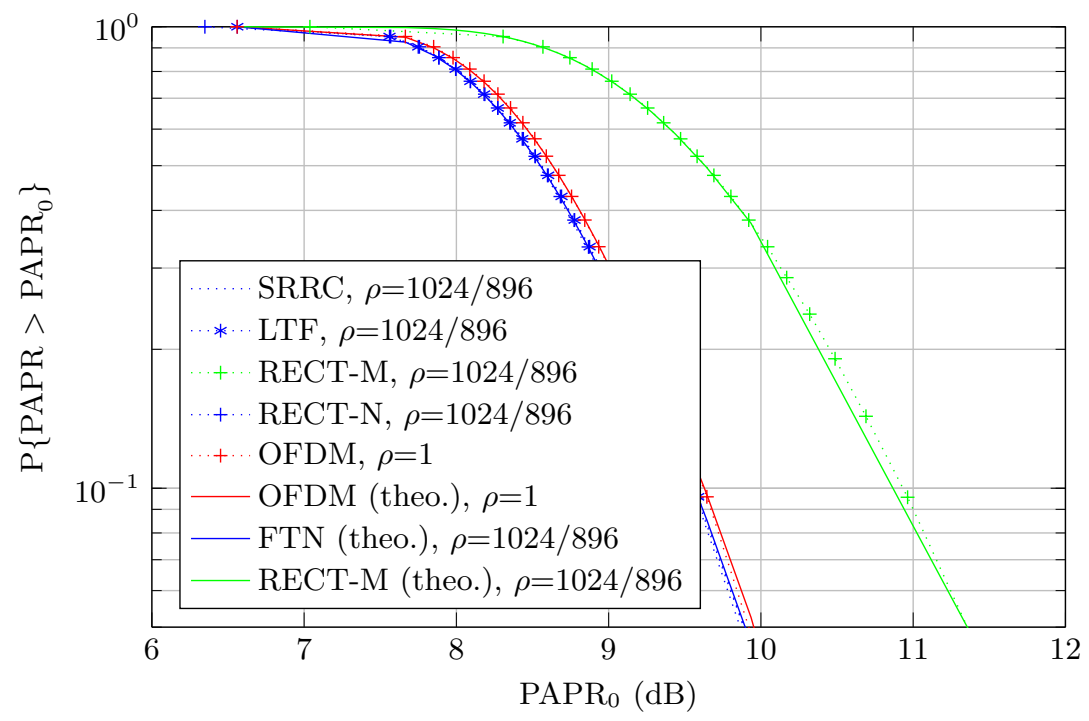

Fig. 5. Discrete-time PAPR comparison for $M=1024$, a quadrature phase shift keying (QPSK) constellation, $\rho \approx 1.14$, and various prototypes. 


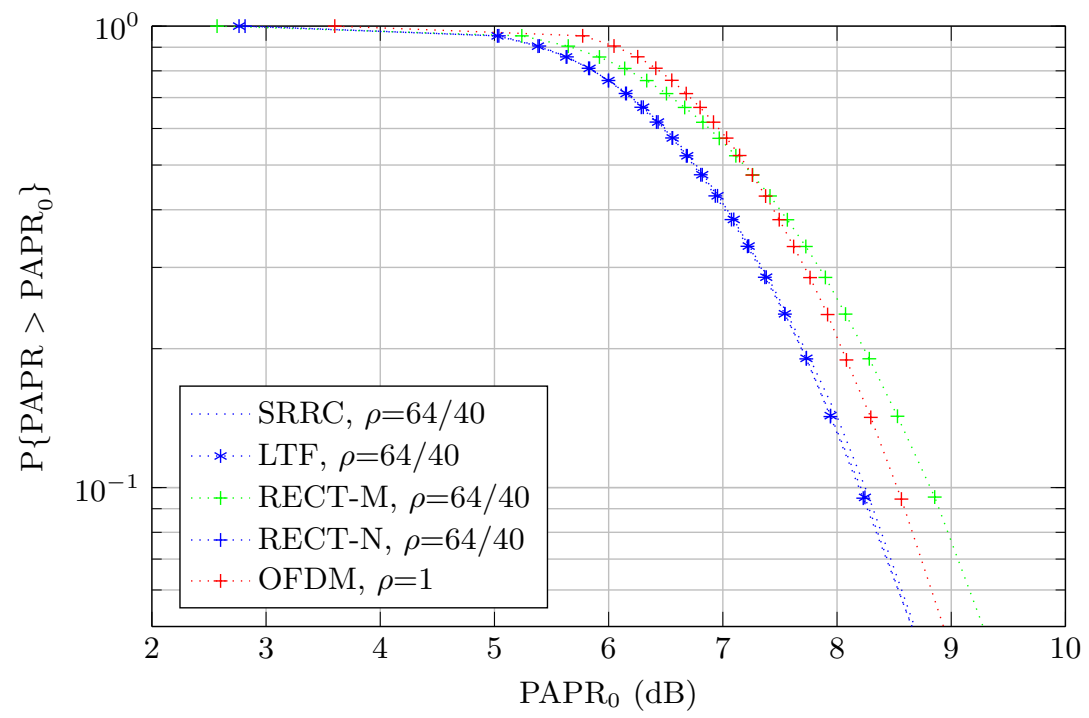

Fig. 6. Continuous-time PAPR comparison for $M=64$, a quadrature phase shift keying (QPSK) constellation, $\rho=1.6$, and various prototypes.

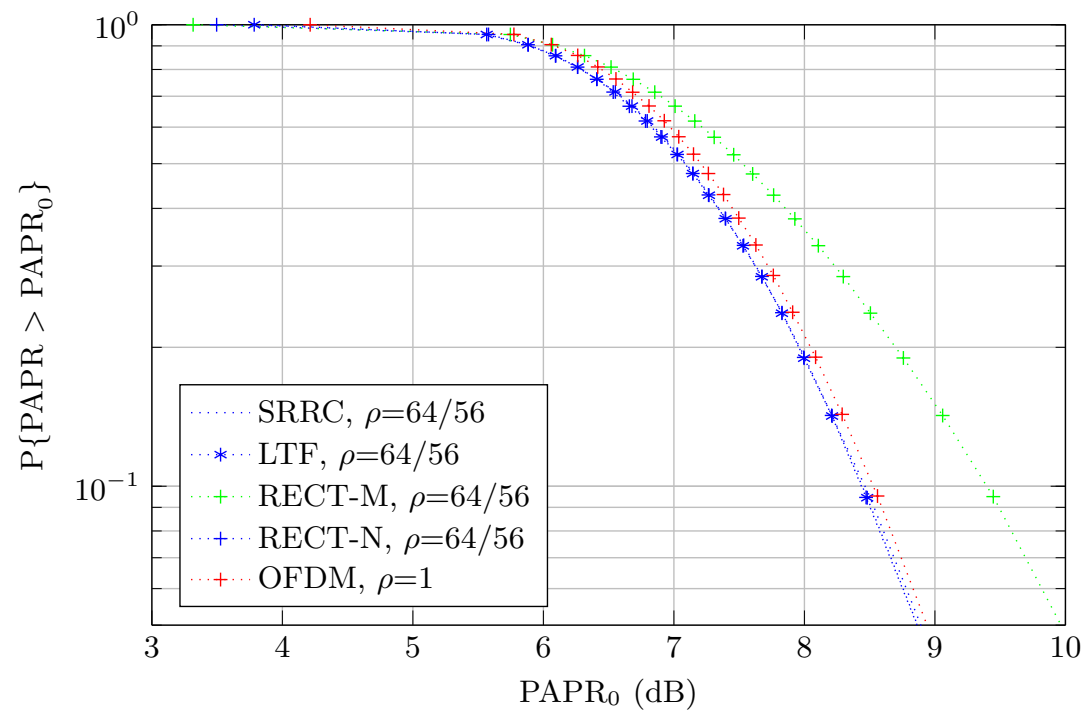

Fig. 7. Continuous-time PAPR comparison for $M=64$, a quadrature phase shift keying (QPSK) constellation, $\rho \approx 1.14$, and various prototypes. 


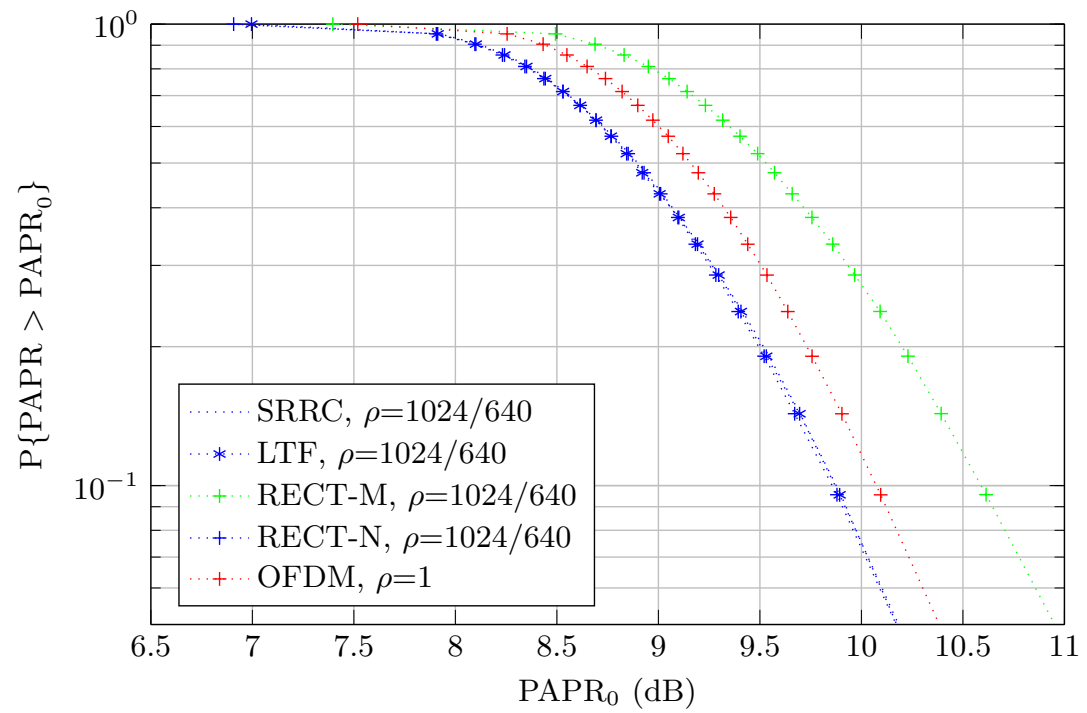

Fig. 8. Continuous-time PAPR comparison for $M=1024$, a quadrature phase shift keying (QPSK) constellation, $\rho=1.6$, and various prototypes.

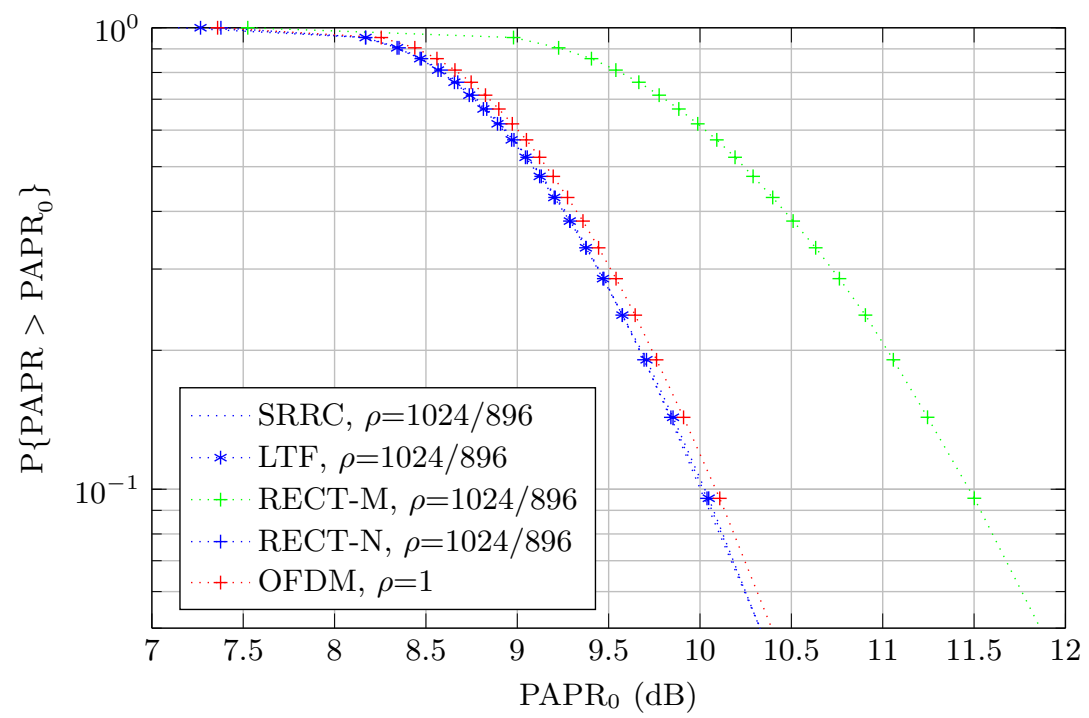

Fig. 9. Continuous-time PAPR comparison for $M=1024$, a quadrature phase shift keying (QPSK) constellation, $\rho \approx 1.14$, and various prototypes. 


\section{Conclusion}

In this article, we have firstly shown that PAPR distribution as derived in [11] remains accurate in the case of practical critically sampled FTN multicarrier systems. Furthermore, optimum PAPR performance can be obtained in the FTN case provided that (i) pulse shapes are chosen as tight frames and (ii) a sufficiently large number of subcarriers is considered. Interestingly, at a given number of subcarriers, FTN multicarrier systems based on tight frames achieve better PAPR performance than traditional OFDM.

An open issue concerns the case where the multicarrier signal samples are not independent. Hypothesis of independence is clearly not relevant when dealing with an oversampled transmitted multicarrier signal. Consequently, the PAPR distribution model derived in $[11,12]$ does not hold anymore. As future work, one could investigate the conditions under which the transmitted samples are independent. A second step could involve the derivation of more general PAPR models (or instantaneous transmitted power [19]) allowing to confirm (or revoke) the constraints to be fulfilled by optimum pulse shapes.

\section{References}

1. B. Sklar, "Rayleigh fading channels in mobile digital communication systems. i. characterization," IEEE Commun. Mag., vol. 35, no. 9, pp. 136-146, 1997.

2. E. Haas, "Aeronautical channel modeling," IEEE Trans. Veh. Technol., vol. 51, no. 2, pp. 254-264, 2002.

3. D. W. Matolak, "Channel modeling for vehicle-to-vehicle communications," IEEE Commun. Mag., vol. 46, no. 5, pp. 76-83, May 2008.

4. W. Kozek and A. F. Molisch, "Nonorthogonal pulseshapes for multicarrier communications in doubly dispersive channels," IEEE J. Sel. Areas Commun., vol. 16, no. 8, pp. 1579-1589, 1998.

5. P. Jung, "Pulse shaping, localization and the approximate eigenstructure of LTV channels (special paper)," in Wireless Commun. and Networking Conf. WCNC 2008. IEEE, 2008, pp. 1114-1119.

6. D. Roque and C. Siclet, "Performances of weighted cyclic prefix OFDM with lowcomplexity equalization," IEEE Commun. Lett., vol. 17, no. 3, pp. 439-442, 2013.

7. F. Rusek and J. Anderson, "The two dimensional Mazo limit," in Int. Symp. on Inform. Theory, ISIT. IEEE, sept. 2005, pp. $970-974$.

8. — - "Multistream faster than Nyquist signaling," IEEE Trans. Commun., vol. 57, no. 5, pp. $1329-1340$, may 2009.

9. A. Marquet, C. Siclet, and D. Roque, "Analysis of the faster-than-Nyquist optimal linear multicarrier system," Comptes Rendus Physique, Feb. 2017.

10. S. H. Han and J. H. Lee, "An overview of peak-to-average power ratio reduction techniques for multicarrier transmission," IEEE Wireless Commun., vol. 12, no. 2, pp. 56-65, April 2005.

11. A. Skrzypczak, P. Siohan, and J. P. Javaudin, "Analysis of the peak-to-average power ratio of the oversampled OFDM," in Int. Conf. on Acoustics Speech and Signal Process. (ICASSP), vol. 4. IEEE, May 2006, pp. IV-IV. 
12. M. Chafii, J. Palicot, and R. Gribonval, "Closed-form approximations of the peak-to-average power ratio distribution for multi-carrier modulation and their applications," EURASIP J. on Advances in Signal Process., vol. 2014, no. 1, p. 121, 2014. [Online]. Available: http://dx.doi.org/10.1186/1687-6180-2014-121

13. C. Siclet, P. Siohan, and D. Pinchon, "Oversampled orthogonal and biorthogonal multicarrier modulations with perfect reconstruction," in Int. Conf. on Digital Signal Process., vol. 2. IEEE, 2002, pp. 647-650 vol.2.

14. O. Christensen, Frames and bases: An introductory course. Birkhauser, 2008.

15. H. Nyquist, "Certain topics in telegraph transmission theory," Trans. of the American Institute of Electrical Engineers, vol. 47, no. 2, pp. 617-644, 1928.

16. C. Siclet, D. Roque, H. Shu, and P. Siohan, "On the study of faster-than-Nyquist multicarrier signaling based on frame theory," in Int. Symp. on Wireless Commun. Syst., ISWCS. IEEE, Aug 2014, pp. 251-255.

17. W. Chongburee, "Analysis of power spectral density of digitally-modulated combined pulse trains," in Annual Conf. on Elect. Eng./Electron., Comput., Telecommun. and Information Technology, ECTI, 2005.

18. D. Pinchon and P. Siohan, "Closed-form expressions of optimal short PR FMT prototype filters," in Global Telecommun. Conf., GLOBECOM. IEEE, Dec 2011, pp. $1-5$.

19. C. Ciochina, F. Buda, and H. Sari, "An analysis of OFDM peak power reduction techniques for wimax systems," in Int. Conf. on Commun., ICC, vol. 10. IEEE, June 2006, pp. 4676-4681. 